\title{
Candidiasis- The Most Common Fungal Infection of Oral Cavity
}

\author{
Neha Aggarwal ${ }^{1}$, Sumit Bhateja* ${ }^{* 1}$, Geetika Arora ${ }^{2}$ and Tehmeena Yasmin ${ }^{1}$ \\ ${ }^{1}$ Department of Oral Medicine \& Radiology, Manav Rachna Dental College, India \\ ${ }^{2}$ Department of Public Health Dentistry, Inderprastha Dental College, India
}

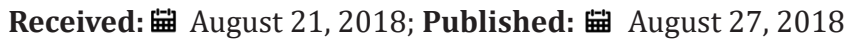

*Corresponding author: Sumit Bhateja, Department of Oral Medicine \& Radiology, Manav Rachna Dental College, India

\begin{abstract}
Oral candidiasis is an opportunistic infection of the oral cavity caused by the overgrowth of Candida species, usually of C. albicans. Candida species are present as commensal organisms of the oral micro biota in about $20-60 \%$ of normal human population. The present article discusses about most common fungal infection of the oral cavity.
\end{abstract}

Keywords: Candidosis; Candida Albicans; Oral cavity

Abbreviations: APECED: Autoimmune Polyendocrinopathy Candidosis Ectodermal Dystrophy; AIDS: Acquired Immune Deficiency Syndrome; HIV: Human Immune Deficiency Virus; SCID: Severe Combined Immunodeficiency Syndrome; CHS: Chronic Hyperplastic Candidosis; MRG: Median Rhomboid Glossitis

\section{Introduction}

The genus Candida belongs to yeasts. It is also the most common cause of opportunistic mycoses worldwide. It is a frequent colonizer of human skin and mucous membranes [1]. Candida is a member of normal flora of skin, mouth, vagina, and bowel. In addition to being a colonizer and a pathogen, it is found in the environment, particularly on leaves, flowers, water and soil. The genus Candida includes around 154 species. Among these, six are most frequently isolated in human infections. While Candidaalbicans is the most abundant and significant species, Candida tropicalis, Candidaglabrata, Candida parapsilosis, Candida krusei, and Candida lusitaniae are also isolated as causative agents of Candida infections. Importantly, there has been a recent increase of infections due to non-albicans Candida spp., such as Candida glabrata and Candida krusei. In the mouth, the primary site where C. albicans is located is the dorsum of tongue, while other places such as tooth surfaces covered with plaque are less commonly colonized.

\section{Etiology and Predisposing Factors [1,2]}

Among the 150 Candida species known today, the most common etiological microorganism of oral candidosis is C. albicans (Figure 1). This, however, does not preclude the other human pathogenic species from being direct causative agents in some cases of oral candidosis, namely C. parapsilosis, C. tropicalis, C. glabrata, C. krusei, C. pseudotropicalis, or even C. dubliniensis, which has first more recently been associated with oral candidosis in HIVinfected patients. There are many factors which predispose to the development of oral candidosis. These can act either by enhancing the growth and colonization of candidal yeast or suppressing the immune system of the host or both. Some factors, which are considered common, are detailed further below.

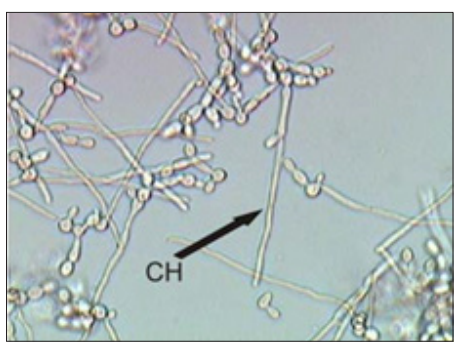

Figure 1: Showing hyphae of C. albicans, $\mathrm{CH}$.

Prostheses: Dental prosthesis, especially if they are ill-fitting and accompanied with poor oral hygiene, may become a substrate for candidal growth. Constant physical irritation can cause local microscopic breaches in the oral mucosa, which provide an entrance route to the fungus. It has been noticed that salivary yeast counts are much higher in patients wearing full dentures than in dentate subjects. Since dental prostheses may restrict the diffusion of oxygen and flow of saliva to the underlying tissue, a local stagnant environment with a low $\mathrm{P}^{\mathrm{H}}$ and oxygen content may be produced.

Epithelial Alterations: Intact oral mucous membrane provides an effective physical barrier against ingress of fungal or bacterial cells. When the turnover rate of epithelial cells alters, e.g. due to radiation therapy or anticancer medication, the integrity of 
oral epithelial seal is impaired, which predisposes the mouth to candidal infection.

Endocrine Disorders: Deficiencies of certain hormones predispose to the emergence of oral candidosis e.g. diabetes mellitus, hypothyroidism, hypoparathyroidism, hypoadenalism and Addison's disease. More commonly, the mucocutaneous form of candidosis has been associated with multiple endocrine disorders where the triad of chronic mucocutaneous candidosis, hypoparathyroidism and adrenocortical failure comprise a compex condition known as APECED (Autoimmune PolyendocrinopathyCandidosis-Ectodermal Dystrophy). Studies have found that Candida species also in asymptomatic patients are more common in the oral cavity of diabetic patients than in healthy people.

Infectious and Immunologic Disorders: The cell-mediated and humoral immunity are of paramount importance in protecting the oral mucosa against candidosis. Since Candida species are opportunistic pathogens, fungal infections are common in patients whose immune system is compromised, e.g. acquired immune deficiency syndrome (AIDS) in which more than 90\% of affected patients at some stage of the disease develop oral candidosis. Human immune deficiency virus (HIV) alters cell-mediated immunity so that T-cell function becomes impaired. Oral candidosis in HIV + ve patients can take up a variety of expressions ranging from pseudomembranous, atrophic and hyperplastic, all of which resemble clinically the lesions seen in noninfected individuals. HIVrelated oral candidosis can also extend beyond the frontiers of the oral cavity to involve other organs such as the esophagus and the trachea, which will cause dysphagia and restrosternal discomfort. Severe combined immunodeficiency syndrome (SCID) is another condition characterized by defects in the cell-mediated and humoral immune reactions. Chronic mucocutaneous candidosis is often noticed in patients with SCID. Patients on immunosuppressive drugs, e.g. following organ transplantation, are also susceptible to oral candidosis.

\section{Classification of Candidiasis}

[1] Pseudomembranous type

Atrophic (erythematous)-antibiotic stomatitis

\section{Atrophic}

Denture sore mouth

Angular cheilitis

Median rhomboid glossitis

\section{Hypertrophic/Hyperplastic}

Candida leukoplakia

Papillary hyperplasia of palate

Median rhomboid glossitis

\section{Multifocal Syndrome Associated}

Familial endocrine neoplasia syndrome

Myositis

\section{Localized}

\section{Generalized}

\section{Clinical Presentation}

\section{Acute Pseudomembranous Candidosis (Thrush)}

This type is characterized by the presence of extensive white superficial patches. These curd-like patches are nothing but pseudo membranes consisting of desquamated epithelial cells, fibrin and fungal hyphae. The pseudo membranes can be wiped off leaving an erythematous base. This lesion can be found anywhere in the oral cavity, most often on the dorsum of tongue or on labial and buccal mucosae. Young infants and old people are frequently affected.

\section{Acute Atrophic Candidosis}

This form of candidosis is also called acute erythematous candidosis. It is not as frequent as thrush except perhaps after the use of broad spectrum antibiotic, but when it occurs it causes painful burning sensation of the mouth. These lesions are characterized by red, atrophic changes occurring most commonly on the palate and tongue. Loss of tongue papillae (depapillation) leads to a bright red appearance and soreness. This clinical type of candidosis is commonly seen in HIV infections and after use of corticosteroids or broad-spectrum antibiotics.

\section{Chronic Atrophic Candidosis (Denture Stomatitis)}

This particular form is characterized by the presence of chronic erythematous and edematous lesions, which range from small to large. It affects at some stage about half of all complete denture wearers (Budtz-Jorgensen 1990). As the synonym suggests, it is associated with ill-fitting and/or poorly cleaned dentures. Therefore, it tends to be found exclusively in the denture-wearing areas, especially the palate. Clinically, it manifests as bright red, somewhat velvety to pebbly surface.

\section{Candida Associated Angular Cheilitis}

Since angular cheilitis is commonly associated with denture stomatitis, it belongs to the same category as chronic atrophic candidosis but has a different location. As the name points, it affects the angles of the oral cavity (commissures) and it starts as fissures which allow pooling of saliva and incubation of candidal cells leading to erythematous lesions which may encrust orerode and hence turn painful.

\section{Median Rhomboid Glossitis}

Median rhomboid glossitis is a chronic symmetrical depapillated area of the tongue anterior to the circumvallate papillae. The region of papillary atrophy is usually elliptical or rhomboid in shape and situated at the midline of the tongue.

\section{Chronic Hyperplastic Candidosis}

Explained in detail below

\section{Chronic Mucocutaneous Candidosis}

This term, which comprises an array of clinical manifestations, is applied when the candidal infection involves organs other than the mouth e.g. skin, nail beds, vagina etc. Four subtypes have been recognized, associated with familial susceptibility; endocrinopathy; 
both familial susceptibility and endocrinopathy; and onset later than 10 years of age.

\section{Chronic Hyperplastic Candidiasis (CHC)}

[3] Lehner first reported the presence of candidal infection in an oral leukoplakia and called it candidal leukoplakia Lehner 1967. Despite the confusion it may cause, most histopathologists prefer to use the term "chronic hyperplastic candidosis" instead of candidal leukoplakia. $\mathrm{CHC}$ is considered the most important clinical form of oral candidosis due to its association with the development of malignancy at the lesion site. This tendency should prompt the oral diagnostician to consider an already-forming real cancer as a differential diagnosis (Figure 2).

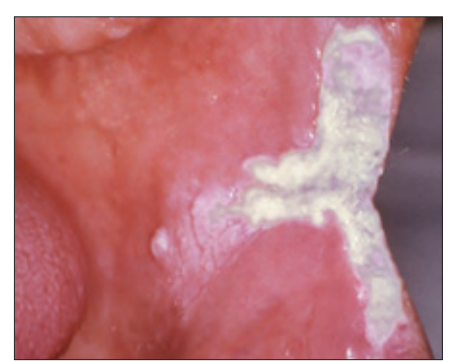

Figure 1: Chronic plaque type Candidiasis. S.

\section{Host Factors in Candidal Leukoplakia/Chronic Hyperplastic Candidosis}

It is well-known that Candida species are normal commensals in up to $50 \%$ of the healthy population. It is therefore conceivable that many local and systemic host factors may operate in vivo to facilitate the conversion of this harmless commensal to a pathogenic organism. These are adequately dealt with elsewhere in relation to the many clinical variants of oral candidosis4. In the following section, we attempt to clarify the role of these factors in the pathogenesis of $\mathrm{CHC}$.

\section{Local Predisposing Factors}

It is likely that a major element leading to the initiation of CHC is a breach of the integrity of the host oral mucosa. Trauma to the mucosa from natural as well as artificial teeth is relatively common. Epithelial changes of the oral mucosa, such as atrophy, hyperplasia, and dysplasia, may compromise the mucosal barrier and may facilitate candidal invasion, especially in the event of epithelial atrophy (Table 1). Reduced salivary flow rate in diseased states such as Sjögren's syndrome, or during cytotoxic therapy and radiation therapy, has been shown to favor increased oral carriage of Candida and a concomitant increase in candidal infection. Although no definite link between xerostomia and CHC has been reported, it is conceivable that $\mathrm{CHC}$ may be precipitated with other variants of candidosis in xerostomic patients, provided that conducive factors co-exist. Apart from the quantitative changes in salivary flow discussed above, qualitative changes of saliva, such as its glucose content and $\mathrm{pH}$, are also factors that may influence oral candidal colonization and probably indirectly affect the genesis of CHC [4] High glucose content of saliva and its low pH have been shown by several workers to favor oral candidal colonization [5] tobacco smoking and chewing habits.

Table 1: Summary of factors predisposing to oral candidosis.

\begin{tabular}{|c|c|c|c|}
\hline \multicolumn{2}{|c|}{ Promote the Growth of Yeasts } & \multicolumn{2}{|c|}{ Suppress the Host Defense } \\
\hline Intrinsic & Extrinsic & Local & Systemic \\
\hline Imbalance of oral microflora & High carbohydrate diet & Hyposalivation & $\begin{array}{l}\text { Extremity of age, i.e. infancy/ } \\
\text { senility }\end{array}$ \\
\hline Low $p^{H}$ & Dental Prostheses & Epithelial changes & $\begin{array}{l}\text { Immunosuppressive infections } \\
\text { e.g. AIDS }\end{array}$ \\
\hline Poor oral hygiene & & Broad spectrum antibiotics & $\begin{array}{l}\text { Immunosuppressive therapy e.g. } \\
\text { Cortisone }\end{array}$ \\
\hline \multirow[t]{3}{*}{ Already existing lesions e.g. Leukoplakia } & & $\begin{array}{c}\text { Malnutrition or Malabsorption, e.g. iron } \\
\text { deficiency }\end{array}$ & Endocrinopathies e.g. Diabetes \\
\hline & & Radiation therapy & pregnancy \\
\hline & & Heavy smoking & \\
\hline
\end{tabular}

\section{Systemic Predisposing Factors [5] \\ Diabetes Mellitus}

Only a minority of patients with candidal leukoplakia have associated medical conditions, including diabetes mellitus. Furthermore, an analysis by the present authors of a large number of reports on oral candidal infections among diabetics reveals that candidal leukoplakia is fairly uncommon in this patient group.

\section{Immunological Aspects}

It was suggested that hyperplastic candidosis could be considered a superficial cellular reaction to the pathogen, which cannot entirely be eradicated by the systemic or local host immune response. As with any other infection, both specific as well as nonspecific immune mechanisms are involved in the defences against human candidal infections. As far as specific immunity against oral candidosis is concerned, both secretory $\operatorname{IgA}$ and cellular immunity might play a role in the protection of the oral mucosal surfaces against candidal infection. Indeed, a markedly increased prevalence of candidal infection can be seen in IgAdeficient individuals.

\section{Nutritional Factors in the Pathogenesis of Candidal Leukoplakia}

Role of iron deficiency shows that oral candidosis may be caused in the deficient individual by at least four mechanisms that render the oral mucosa susceptible to infection by the fungus. Iron deficiency can cause epithelial abnormalities such as 
hyperkeratosis and atrophy through alterations in the kinetics of the rapidly dividing cells of the oral mucosa, which, in turn, result from an impairment of iron-dependent enzyme systems.

\section{Clinical Manifestations}

Chronic hyperplastic candidosis appears as discrete, raised lesions. These hyperplastic, sometimes nodular lesions may be speckled or homogenous and they range in size from small, palpable lesions to large dense plaques. They are most often white or cream colored but occasionally red. The red and speckled lesions have the clinical appearance of numerous white nodules on an erythematous base. They occur on the buccal mucosa, palate, or dorsum of the tongue. These lesions cannot be wiped off. CHC presenting as leukoplakia appears as well-demarcated, palpable, raised lesions that may vary from small translucent whitish areas to large opaque plaques that cannot be rubbed off. Some or all areas of the plaque may have a smooth, homogenously white surface, and if this feature predominates, the lesion is referred to as a homogenous leukoplakia. However, the surface often has erythematous areas intermingled with white areas that, more often than not, possess a nodular characteristic. Such lesions are referred to as nodular or speckled leukoplakia. Although many homogenous lesions are asymptomatic, speckled leukoplakias cause intermittent soreness or discomfort.

As mentioned earlier, the most common site for these lesions is the buccal mucosa, especially the commissural areas. The palate and tongue may also be involved, although less frequently, with the former being affected relatively more often. Not uncommonly, the commissural lesions of CL tend to be associated with angular cheilitis. Indeed, in about one-third of candidal leukoplakias, other forms of oral candidosis are found to coexist. These are Candida-associated denture stomatitis, angular cheilitis, median rhomboid glossitis (MRG), and an oval or circular erythematous lesion on the palate in the area corresponding to that of MRG. The term "chronic multifocal candidosis" has been used to describe this tetrad, the constant member of the group being commissural candidal leukoplakia [6]. Histopthaologically candidal cells, yeasts and hyphae, are seen on the uppermost tissue surface, and when they invade the epithelium (hyphal and pseudohyphal invasion), they rarely penetrate beyond the spinous layer. The lesions show hyperparakeratotic or hyperorthokeratotic epithelium with irregular separation and epithelial hyperplasia. There is a higher mitotic activity, but it is restricted to the basal and suprabasal layers of the epithelium [7]. Typical feature is the presence of micro abscesses, which are collections of polymorphonuclear leukocytes in the epithelium. Lamina propria contains an inflammatory cell infiltrate composed mostly of lymphocytes, macrophages and plasma cells.

\section{Diagnosis}

Exfoliative cytology, culture or tissue biopsy. Exfoliative cytology is performed by scraping superficial cells to samples. A swab culture should be taken on the undersurface of a denture when denture stomatitis is suspected. If the candidal infection is thought to have already invaded the tissue, sampling some tissue in form of a biopsy should be considered. Hematological screening might be useful since up to some $40 \%$ of patients with oral candidosis may have some haematological abnormalities [8].

\section{Management}

\section{Elimination of the Predisposing Factors}

Once a diagnosis of oral candidosis is assured, the first line of treatment should aim to eliminate or alleviate any dental and/ or medical factors which contribute to the occurrence of candidal infection in the oral cavity. The oral diagnostician may find it necessary to refer his/her patient, in case there is suspicion of any pertinent medical condition, to their physicians to seek for advice. Patient cooperation is sometimes crucial especially when the predisposing factors are social rather than medical e.g. smoking. One of the most important factors contributing to chronic hyperplastic candidosis is smoking; therefore, cessation of smoking is mandatory to relieve the disease. Chronic atrophic candidosis is mostly associated with edentulous patients who wear dentures; therefore, patient education and motivation to cleanse their dentures regularly and apply the oral hygiene measures is necessary to restore the infected edentulous mucosa to normalcy. If patients with acute atrophic candidosis are on broad spectrum antibiotics or corticosteroid, discussion with their physicians about the possibility of withdrawal or substitution of the medicine could resolve the problem.

\section{Antifungal Treatment}

Antifungal therapy has been used successfully in the management of oral candidosis. Prior to prescription of any antifungal agents, advising the patient to gargle with a physiological saline solution helps to decrease the oral fungal counts and thus soothe the associated symptoms. Pharmacological treatment of oral candidosis should be tailored to the individual patients according to their current medical status and severity of infection. Antifungal agents are available in different forms (i.e. gels, ointment, creams, suspension, lozenges, and tablets) and the dentist should manage to select the proper form upon writing a prescription. There are many drug categories available to treat the different clinical presentations of candidosis, summarized as follows:

a) Polyenes: The candidal cell wall is composed of many layers, the innermost of which is the plasma membrane. Polyenes are potent agents which act by binding to the sterol part of the candidal cell membrane and disrupt its osmotic integrity leading to leakage of essential ions e.g. potassium and magnesium. This category includes nystatin and amphotericin B. Nystatin, which is of bacterial origin, is the drug of choice for treatment of oral candidosis. It should be prescribed cautiously in patients with uncontrolled diabetes or xerostomia. Amphotericin B can be used to treat superficial candidosis i.e. Fungizone (oral), or more wide-spread systemic involvement i.e. Ambisome (Intravenous).

b) Antimetabolites: This group contains only one agent: flucytosine (5-Fluorocytosine). 
c) Azoles: This class includes two major categories: imidazole (e.g. clotrimazole, miconazole, econazole, ketoconazole) and triazole (e.g. Fluconazole, Itraconazole).

d) Glucan Synthesis Inhibitors: These (e.g. caspofungin) act by inhibiting the essential component of the fungal cell wall, glucan.

Miscellaneous Agents: There is only one drug available of this class of current use i.e. griseofulvin which exerts its effect through disrupting the mitotic spindle's.s

\section{References}

1. Greenberg MS, Glick M Burket's (2005) Oral Medicine Red and white lesions of the oral cavity. Delhi, India: BC decker Inc, Elsevier, (10 ${ }^{\text {th }}$ Edn $)$, pp 85-125.

2. Ongole R, BN Parveen (2013) Textbook of Oral Medicine Oral Diagnosis and Oral Radiology. Red and white lesions. Chennai, India: Elsevier $\left(2^{\text {nd }}\right.$ Edn.), Pp. 133-173.

ISSN: 2574-1241

DOI: 10.26717/BJSTR.2018.08.001649

Sumit Bhateja. Biomed J Sci \& Tech Res

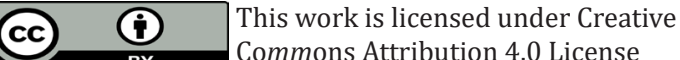

Submission Link: https://biomedres.us/submit-manuscript.php
3. Sitheeque, MA, LP Samaranayake (2003) Chronic hyperplastic candidosis/candidiasis (candidal leukoplakia). Crit Rev Oral Biol Med14(4): 253-267.

4. Andriole VT, Hasenclever HF (1962) Factors influencing experimental candidiasis in mice. I. Alloxan diabetes. Yale J Biol Med 35(1): 96-142.

5. Arendorf TM (1984) Factors affecting oral candidal colonisation in health and disease. Br Dent J 147: 267-272.

6. Cawson RA, Lehner T (1968) Chronic hyperplastic candidosis-candidal leukoplakia. Br J Dermatol 80(1): 9-16.

7. Cernea P, Crepy C, Kuffer R, Mascaro JM, Badillet G, Marie JL (1965) Aspects peu connus de candidosis buccales. Les Candidases à foyers multiples de la cavité buccale. Rev Stomatol (Paris) 66: 103-138

8. Challacombe SJ (1986) Haematological abnormalities in oral lichen mplanus, candidiasis, leukoplakia and non-specific stomatitis. Int J Oral Maxillofac Surg 15(1): 72-80.

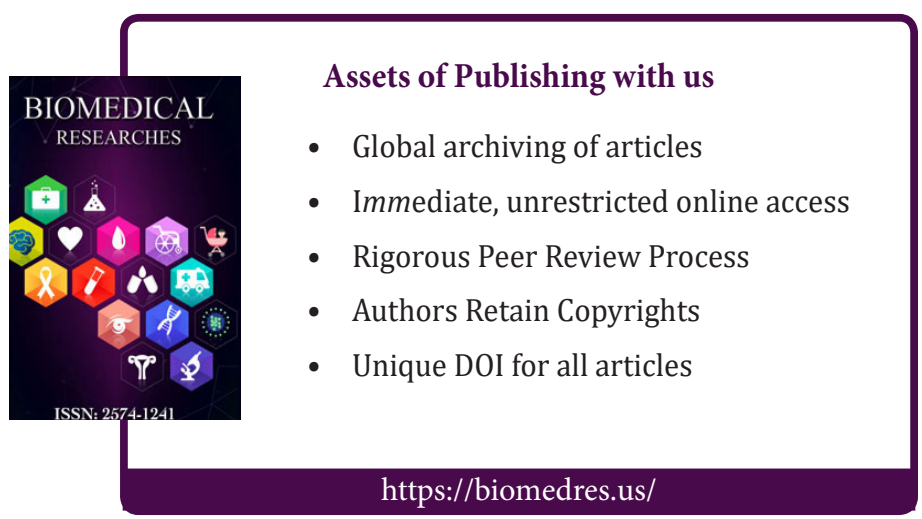

